\title{
Differential gene body methylation and reduced expression of cell adhesion and neurotransmitter receptor genes in adverse maternal environment
}

\author{
J-e Oh ${ }^{1}$, N Chambwe ${ }^{2}$, S Klein ${ }^{1}$, J Gal ${ }^{3}$, S Andrews ${ }^{2}$, G Gleason ${ }^{1}$, R Shaknovich ${ }^{4}$, A Melnick ${ }^{4}$, F Campagne ${ }^{2}$ and M Toth ${ }^{1}$
}

\begin{abstract}
Early life adversity, including adverse gestational and postpartum maternal environment, is a contributing factor in the development of autism, attention deficit hyperactivity disorder (ADHD), anxiety and depression but little is known about the underlying molecular mechanism. In a model of gestational maternal adversity that leads to innate anxiety, increased stress reactivity and impaired vocal communication in the offspring, we asked if a specific DNA methylation signature is associated with the emergence of the behavioral phenotype. Genome-wide DNA methylation analyses identified $2.3 \%$ of CpGs as differentially methylated (that is, differentially methylated sites, DMSs) by the adverse environment in ventral-hippocampal granule cells, neurons that can be linked to the anxiety phenotype. DMSs were typically clustered and these clusters were preferentially located at gene bodies. Although CpGs are typically either highly methylated or unmethylated, DMSs had an intermediate (20-80\%) methylation level that may contribute to their sensitivity to environmental adversity. The adverse maternal environment resulted in either hyper or hypomethylation at DMSs. Clusters of DMSs were enriched in genes that encode cell adhesion molecules and neurotransmitter receptors; some of which were also downregulated, indicating multiple functional deficits at the synapse in adversity. Pharmacological and genetic evidence links many of these genes to anxiety.
\end{abstract}

Translational Psychiatry (2013) 3, e218; doi:10.1038/tp.2012.130; published online 22 January 2013

\section{Introduction}

A significant factor in the development of psychiatric disorders, including anxiety, depression, autism and attention deficit hyperactivity disorder (ADHD) is the environment, both prenatally and during early postnatal life. ${ }^{1,2}$ Early life adversity, such as maternal stress, maternal infection (for example, immune activation during pregnancy) ${ }^{3}$ and maternal separation during early postnatal life, ${ }^{4}$ and its behavioral consequences on the offspring can be reproduced in nonhuman primates and rodents. Although many genetic tools are available for the mouse, establishing early life adversity paradigms with robust and reproducible behavioral outcomes is challenging in this species. ${ }^{5,6}$ We have recently developed a mouse model of maternal adversity, which is based on a deficit in the maternal $5-\mathrm{HT}_{1 \mathrm{~A}}$ receptor $(\mathrm{R})$ and which causes innate anxiety, increased stress reactivity and impaired vocal communication in the offspring. ${ }^{7,8}$ This model has construct validity because reduced binding of $5-\mathrm{HT}_{1 \mathrm{~A}} \mathrm{R}$ has been found in depression, including peri/postpartum depression, a condition that can represent early life adversity for the offspring. ${ }^{7,9}$ This model was developed on the outbred Swiss Webster background (a strain often used in behavioral experiments), to avoid the possible contribution of homozygous genetic variants in inbred strains to behavioral phenotypes. ${ }^{10}$ The unique feature of our maternal adversity model is that the initial trigger is well defined (for example, maternal receptor deficit as opposed to more complex paradigms such as maternal stress and inflammation) and this gives a foothold from which to investigate the underlying molecular mechanism. A partial receptor deficit (heterozygosity, $\mathrm{H}$ ) in the mothers is sufficient to elicit the behavioral abnormalities in the wild-type (WT) offspring ${ }^{7}$ and the behaviors develop independently of the offspring's own receptor genotype (that is, similar effect in the WT and mutant offspring). ${ }^{7}$ Further studies showed that the anxiety of the offspring of $5-\mathrm{HT}_{1 \mathrm{~A}} \mathrm{R}$-deficient mothers is prenatally determined, is not related to maternal care ${ }^{8}$ (a major postnatal factor in anxiety in other models ${ }^{11}$ ), and is linked to the delayed maturation of dentate granule cells (DGCs) in the ventral but not in the dorsal hippocampus (HIP). ${ }^{7}$

The ventral (v)-HIP is part of the distributed and interconnected network of brain regions involved in anxiety. The role for the v-HIP in innate anxiety is supported by the reduced fear and avoidant behavior of rodents following its lesion. ${ }^{12,13}$ Sensory inputs, via the entorhinal cortex, arrive to v-DGCs that are connected to $\mathrm{v}-\mathrm{CA} 3$ and $\mathrm{v}-\mathrm{CA} 1$ neurons, which together form the classical trisynaptic HIP circuit. This circuit sends direct projections to the medial prefrontal cortex and activity in the v-HIP is synchronized with medial prefrontal cortex to produce appropriate defensive and anxiety-related behaviors. ${ }^{14}$ Other connections of V-HIP that are relevant to anxiety include those to the amygdala and the bed nuclei of stria terminalis. ${ }^{15}$

\footnotetext{
${ }^{1}$ Department of Pharmacology, Weill Cornell Medical College, New York, NY, USA; ${ }^{2}$ Department of Physiology and Biophysics and HRH Prince Alwaleed Bin Talal Bin Abdulaziz Alsaud Institute for Computational Biomedicine, Weill Cornell Medical College, New York, NY, USA; ${ }^{3}$ Harold and Margaret Milliken Hatch Laboratory of Neuroendocrinology, The Rockefeller University, New York, NY, USA and ${ }^{4}$ Department of Medicine, Weill Cornell Medical College, New York, NY, USA Correspondence: Dr J-e Oh or Dr M Toth, Department of Pharmacology, Weill Cornell Medical College, New York, NY 10065, USA.

E-mail: jio2002@med.cornell.edu or mtoth@med.cornell.edu

Keywords: CpG island; development; DNA methylation; exon; maternal effect

Received 25 July 2012; revised 12 September 2012; accepted 6 October 2012
} 
Previous studies suggested that adverse maternal environment can produce permanent epigenetic changes in neurons. ${ }^{16-19}$ Among the various epigenetic modifications, CpG methylation is probably the longest lasting, although it can still be dynamically regulated in certain circumstances. ${ }^{20}$ Although DNA methylation assays have long been available, finding methylation changes that underlie environmental effects, including maternal effects, is complicated by the necessity to use homogenous neuronal populations. Indeed, current knowledge on neuronal methylation is largely limited to whole brain ${ }^{21,22}$ and in vitro differentiated neurons/neuronal precursors, ${ }^{23,24}$ although the methylation pattern of mouse DGCs has recently been reported. ${ }^{20}$ Also, the effect of early environmental influences on neuronal DNA methylation has mostly been tested with candidate genes, ${ }^{19,25}$ an approach that does not provide an unbiased survey of epigenetic changes induced by maternal adversity. Here we isolated v-DGCs and performed whole-genome representational analyses by using two assays, Hpall tiny fragment enrichment by ligation-mediated PCR (HELP assay) and enhanced reduced representation bisulfite sequencing (ERRBS), to determine the pattern and developmental dynamics of $\mathrm{CpG}$ methylation produced by maternal adversity. We found that the receptor-deficient maternal effect induced large-scale methylation changes in exons, introns and gene distal areas while changes were underrepresented in promoters. Methylation changes tended to be clustered and the affected genes encode proteins involved in synapse formation and function.

\section{Materials and methods}

Animals. Adult, 10- to 13-week-old Swiss Webster male mice were used in all experiments. Animals were generated as described previously ${ }^{7,8}$ and housed three-five per cage in a room with controlled temperature and a fixed lighting schedule (lights off from 0600 to 1800 hours). Food and water were available ad libitum. All experimental protocols were approved by Research Animal Resource Center at the Weill Cornell Medical College.

Sample dissection and DNA extraction. Frozen brains were sectioned into $200 \mu \mathrm{m}$ slices using a CM3050 cryostat (Leica, Nussloch, Germany) and the ventral dentate gyrus area from the slices was microdissected using a microdissecting knife (FST, Foster City, CA, USA). Ventral dentate gyrus samples from three to five mice from at least three litters were pooled into one tube. DNA extraction was carried out with the QIAGEN Puregene Gentra cell kit (Qiagen, Valencia, CA, USA). DNA was dissolved in $10 \mathrm{~mm}$ Tris (tris[hydroxymethyl]aminomethane)- $\mathrm{HCl}, \mathrm{pH}$ 8.0, and DNA concentration was measured by using NanoDrop ND-1000 (Thermo Scientific, Wilmington, DE, USA).

DNA methylation HELP arrays. The HELP assay was carried out as previously described ${ }^{22,26,27}$ in the Epigenomics Core Facility of the Weill Cornell Medical College. Detailed procedures and further information on data processing are described in Supplementary Material.
DNA methylation sequencing by MassARRAY EpiTYPER. Matrix-assisted laser desorption/ionization-time of flight mass spectrometry-based MassARRAY using EpiTYPER (Sequenom, San Diego, CA, USA) was performed on fragments identified by the HELP assay. First, the DNA was bisulfite converted as previously described ${ }^{28}$ followed by sequencing. MassARRAY primers were designed to cover the Hpall amplifiable fragments and additional 2000-bp upstream and downstream sequences in case the sites at Hpall amplifiable fragments were methylated and the DNA was cut at upstream and/or downstream Hpall sites in the HELP assay. More detailed information including primer sequences is provided in Supplementary Material.

Enhanced reduced representation bisulfite sequencing. In all, $500 \mathrm{ng}$ DNA from each group was processed by the standard RRBS protocol as described ${ }^{29}$ with some modifications described in Supplementary Material. The amplified libraries were sequenced using a 50-bp single end read run on a HiSeq2000 Illumina (San Diego, CA, USA) per manufacturer's recommended protocol. Image capture, analysis and base calling were performed using Illumina's CASAVA 1.7, Illumina. Bisulfite converted reads were mapped to the mouse genome $\mathrm{mm} 9$ with GobyWeb and the last bisulfite plugin. ${ }^{30}$ Methylation rates were estimated with GobyWeb as described in Supplementary Material.

Sample preparation for RNA extraction and RNA-Seq. Mice were perfused with ice-cold saline solution containing $30 \%$ RNAlater (Ambion, Austin, TX, USA) to prevent RNA degradation during microdissection. Brains were rapidly removed, frozen and stored at $-80^{\circ} \mathrm{C}$ until sectioning. Isolation of ventral dentate gyrus was as described above. Ventral dentate gyrus samples from three to five mice from at least three litters were pooled. Details for RNA extraction, RNA-Seq and data analysis are described in Supplementary Material.

Statistical analysis. Data are shown as means \pm s.e. Oneway analysis of variance or Kruskal-Wallis rank sum test was used in the analyses to compare multiple groups followed by least significant difference test and Tukey's post hoc analysis, respectively, to assess statistical significance. Differences between groups were considered to be significant when $P<0.05$.

Data access. Raw data (HELP, RNA-Seq and RRBS) have been deposited into the GEO database and are included in the super series identified by accession number GSE35856.

\section{Results}

Methylation changes in adverse maternal environment in DGCs. As anxiety in the offspring of $5-\mathrm{HT}_{1 \mathrm{~A}} \mathrm{R}$-deficient mothers is associated with the delayed maturation of v-DGCs during early postnatal life, and because genetic interference with v-DGC maturation (by the inactivation of the cyclindependent kinase inhibitor $\mathrm{p} 16^{\text {Ink4a }}$ ) is accompanied by 
a

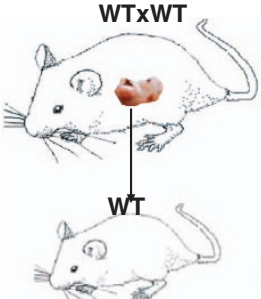

WT(WT)

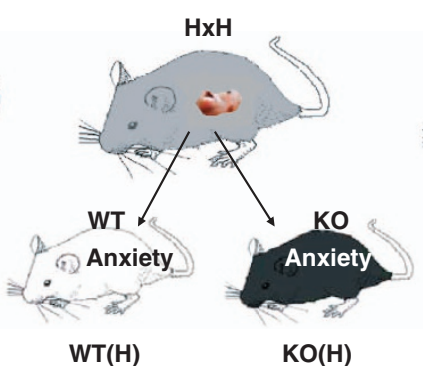

$\mathrm{KO}(\mathrm{H})$

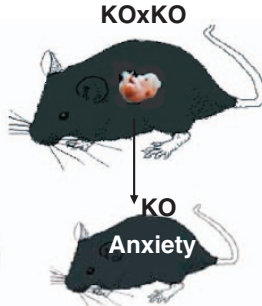

$\mathrm{KO}(\mathrm{KO})$

b

Hypermethylated Hypomethylated
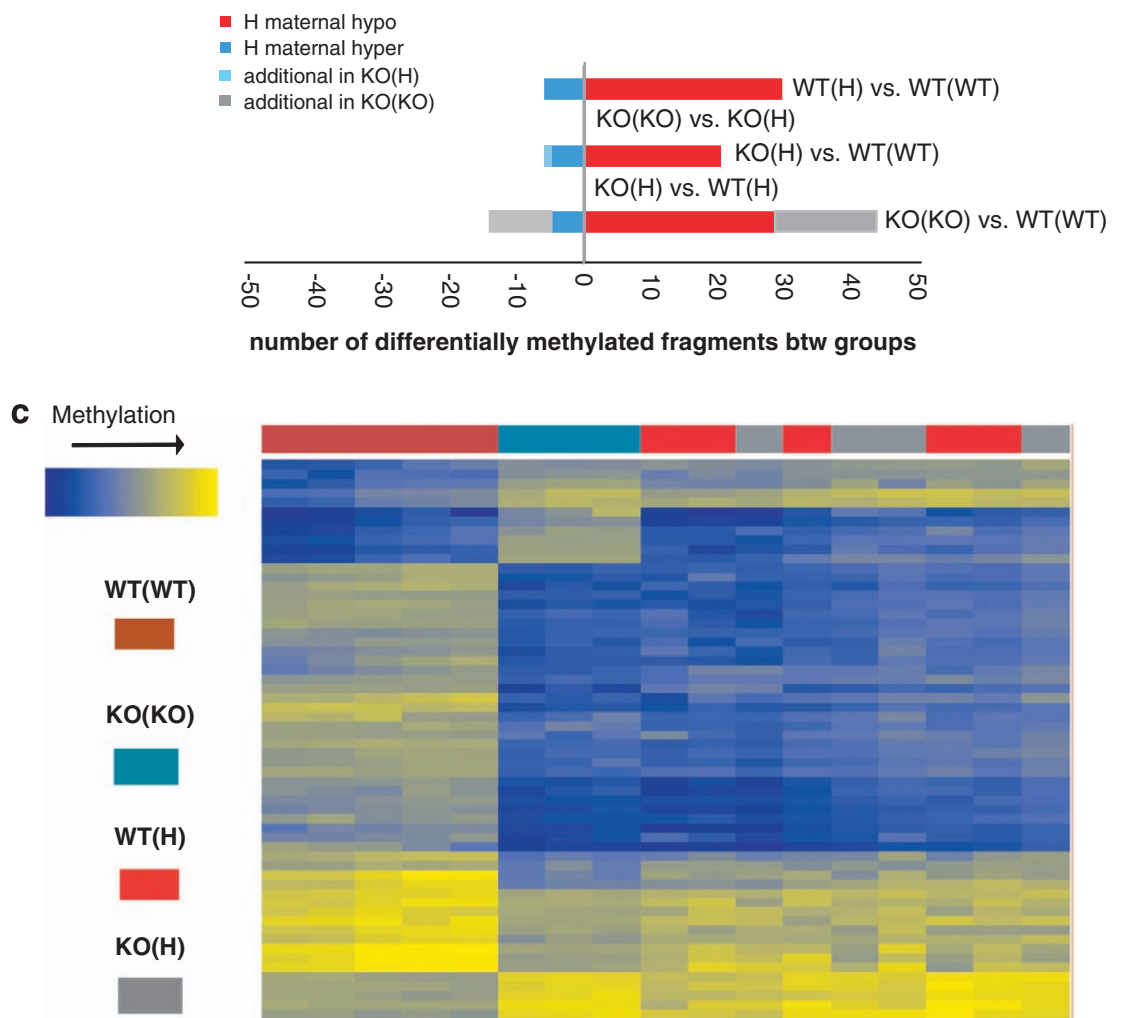

Figure 1 The 5- $\mathrm{HT}_{1 \mathrm{~A}} \mathrm{R}$-deficient maternal environment results in DNA hypo- and hypermethylation in offspring ventral dentate granule cells (DGCs). (a) Wild-type (WT) (and KO) offspring exposed to mutant ( $\mathrm{H}$ or KO) maternal environment exhibit anxiety. (b) Exposure to the $\mathrm{H}$ maternal environment is associated with differential methylation at 35 DNA fragments in the WT offspring and the majority of these differentially methylated regions (DMRs) are also present when the mother and/or the offspring are $\mathrm{KO}$ (KO(H) and $\mathrm{KO}(\mathrm{KO})$. (c) Heat map representation of methylation levels at DMRs across biological replicates of the four groups of animals.

increased anxiety, ${ }^{7}$ we tested v-DGCs for genomic methylation changes associated with the receptor-deficient maternal environment. First, we used the methylation enzyme-based 'HELP' assay, to interrogate CpG methylation at 14392 known RefSeq gene promoters (out of the total of 29716 based on the $\mathrm{NCBI} 37 / \mathrm{mm} 9$ mouse assembly) and $9114 \mathrm{CpG}$ islands (promoter and non-promoter, out of 16026) by using custom arrays. ${ }^{22}$ To identify $\mathrm{H}$-maternal-specific methylation changes, WT offspring of WT mothers (WT(WT)) were compared with the WT offspring of $\mathrm{H}$ mothers $(\mathrm{WT}(\mathrm{H}))$ (Figure 1a). WT(WT) samples were also compared with knockout $(\mathrm{KO})(\mathrm{H})$ and $\mathrm{KO}(\mathrm{KO})$ samples because lack of the receptor in the offspring (in $\mathrm{KO}(\mathrm{H})$ ) and/or the switch of the maternal receptor deficit from partial to complete (in $\mathrm{KO}(\mathrm{H})$ ) had no additional effect on either the delayed DGC development or the anxiety of the offspring, elicited by the $\mathrm{H}$ maternal environment (Figure 1a).

By comparing the methylation level of WT offspring of $\mathrm{H}$ and WT mothers (WT(H) vs WT(WT)), 35 differentially methylated regions (DMRs) were identified (Figure 1b ) out of the 25725 fragments assayed (Benjamini-Hochberg multiple testing, false discovery rate $(\mathrm{FDR})<0.5 \%$, methylation ratio $>1.5$, three litters per replicate, 3-4 biological replicates per group, $r^{2}>0.90$ between replicates). The majority of the WT(H)specific DMRs represented hypomethylation (29 DMRs). When WT and $\mathrm{KO}$ littermates of $\mathrm{H}$ mothers were compared (WT $(\mathrm{H})$ vs $\mathrm{KO}(\mathrm{H})$ ), no DMRs were found indicating that the offspring's own 5- $\mathrm{HT}_{1 \mathrm{~A}} \mathrm{R}$ genotype elicited no methylation 

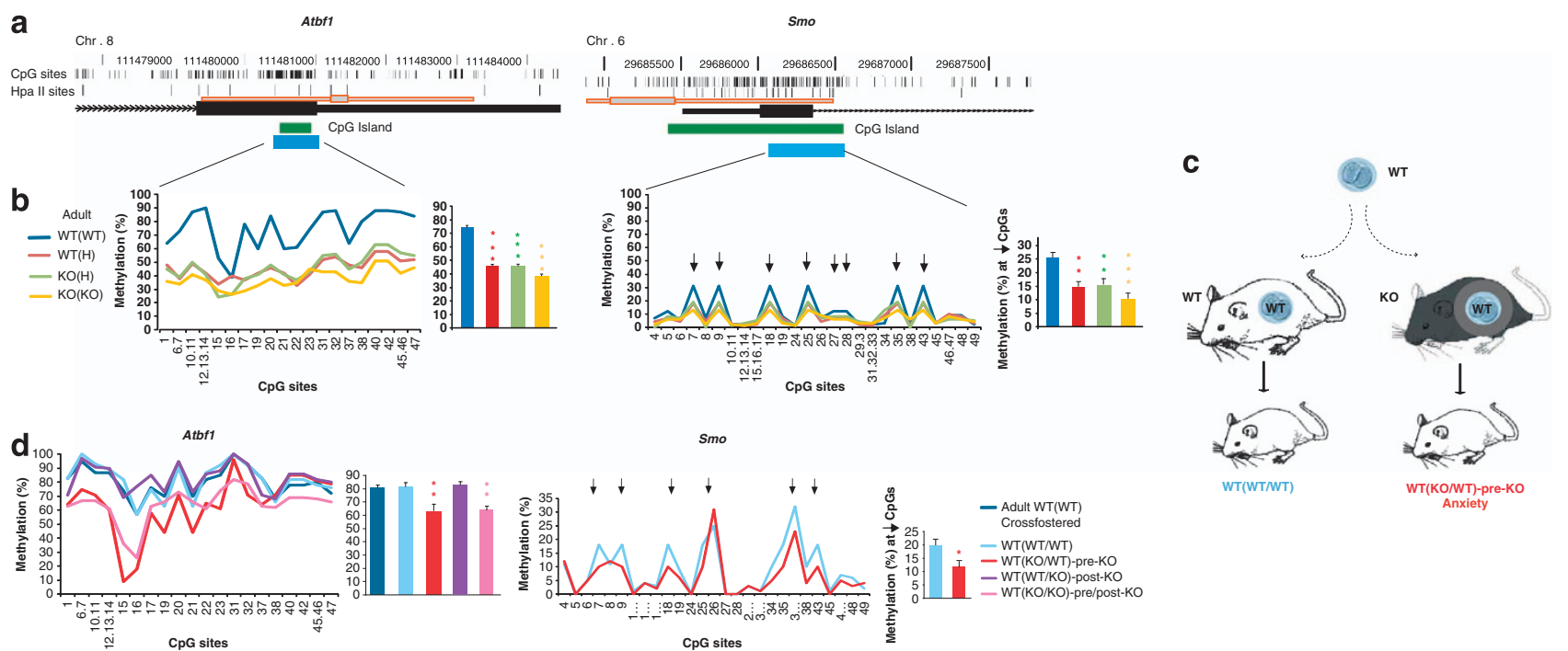

Figure 2 Nucleotide level methylation across differentially methylated regions (DMRs) in two genes. (a) Genomic structure around DMRs. Genomic structure around DMRs. Thick and thin gray bars with red frames indicate the minimal and maximal size of the differentially methylated Hpall tiny fragment enrichment by ligation-mediated PCR (HELP) fragments between Hpall sites. Green boxes represent CpG islands. Blue boxes indicate regions that contained differentially methylated $\mathrm{CpG}$ sites, including $\mathrm{CpGs}$ at Hpall sites, within the HELP fragments. (b) Methylation patterns at individual $\mathrm{CpG}$ sites in various groups of adult animals across DMRs. Similar patterns were reproduced with independent samples. Columns display mean methylation levels with s.e.; analysis of variance (ANOVA) with least significant difference test post hoc: Atbf1, $F_{1,3}=48.00$, $P<0.00001, N=20$; Smo, $F_{1,3}=9.31, P=0.0002, N=8 ;{ }^{*} P<0.05,{ }^{* *} P<0.005,{ }^{* * \star} P<0.0005$ indicate significant differences from the wild-type (WT) offspring of WT mothers (WT(WT)) groups. Statistical analyses were performed by using CpG sites that showed a higher than 10\% methylation level in WT(WT) samples because hypomethylation can be produced by the $\mathrm{H}$ maternal environment only at sites that have a measurable (above the baseline) methylation level when the mother is WT. Although all $\mathrm{CpG}$ sites within the Atbf1 DMR, located at the last exon, fulfilled this criterion, the Smo DMR, located at the first exon (and close to the promoter), showed an overall lower methylation with measurable methylation only at interspersed $\mathrm{CpG}$ sites (indicated by arrows). (c) Embryo transfer and cross-fostering to isolate the effect of the pre- and postnatal receptor-deficient maternal environment on DNA methylation. WT embryos were implanted to KO mothers (and to WT to serve as controls) and newborn pups were then cross-fostered to WT foster mothers. Other combinations of the pre- and postnatal maternal environment are listed in panel (d) but not shown here for simplicity. (d) Offspring exposed to the prenatal KO environment or the combination of the KO pre/postnatal environment show hypomethylation at the Atbf1 and Smo DMRs. Kruskal-Wallis rank sum with Tukey's post hoc. Atbf1: $\times 2=33.4551, P<0.001,{ }^{* \star} P<0.005$ vs WT(WT/WT), $N=20 ;$ Smo: $\times 2=6.60372, P=0.0102,{ }^{*} P<0.05 ; N=6$.

changes that were detectable by HELP (Figure 1b). Importantly, most of the DMRs were also seen when KO offspring of $\mathrm{H}$ and KO mothers were compared with WT(WT) offspring $(\mathrm{KO}(\mathrm{H})$ and $\mathrm{KO}(\mathrm{KO})$-specific DMRs), indicating that similar methylation changes can be elicited by the $\mathrm{H}$ and $\mathrm{KO}$ maternal genotype, independently of the offspring genotype, and that there is a good correspondence between methylation changes and anxiety. However, the $\mathrm{KO}$ as compared with the $\mathrm{H}$ maternal environment caused additional changes in methylation as shown by the heat map representation of all DMRs across the three comparisons (Figure 1c). Approximately half of the DMRs overlapped CpG islands (Supplementary Table 1), a distribution similar to that of the assay. Taken together, the methylation changes, similarly to the delayed V-DGC developmental phenotype and anxiety, ${ }^{7}$ were elicited by either the $\mathrm{H}$ or $\mathrm{KO}$ maternal environment and were independent of the offspring genotype.

Methylation at individual CpG sites within DMRs in adverse maternal environment. To obtain a nucleotide resolution methylation map of maternally induced changes, we performed mass spectrometry-based bisulfite sequencing at and around a number of DMRs. Figures $2 a$ and $b$ display hypomethylation in maternal environment at two DMRs, one located at a distal and another at a proximal exon (expression at the corresponding genes was also downregulated in adverse maternal environment; Supplementary
Table 1). The DMR associated with Atbf1/Zfhx3 (encoding AT-binding transcription factor1, a homeodomain and zincfinger transcription factor ${ }^{31}$ involved in neuronal maturation), ${ }^{32}$ was mapped to a region that includes a strong $\mathrm{CpG}$ island in the last exon (Figure 2a). Sequencing analysis showed relatively high methylation levels throughout this exonic CpG island in adult WT(WT) DGCs, whereas methylation was significantly reduced in DGCs of adult offspring of $\mathrm{H}$ and $\mathrm{KO}$ mothers (Figure $2 \mathrm{~b}$ ). Outside the island, the methylation difference disappeared within $\sim 100$ bps (Supplementary Figure 1). The DMR in Smo (smoothened, co-receptor of sonic hedgehog known to be involved in both patterning and later in neuritogenesis and synaptic differentiation) ${ }^{33,34}$ was associated with a CpG island located at the promoter/ $5^{\prime}$ untranslated region/first exon (Figure 2a). As expected in this promoter proximal region, the overall methylation level was low throughout the island. However, the very low $(0-10 \%)$ methylation was interspersed, specifically across the first exon with small $\sim 30 \%$ peaks in methylation at specific CpGs in WT(WT) neurons that approached only $15-20 \%$ in the neurons of offspring of $\mathrm{H}$ and $\mathrm{KO}$ mothers (Figure $2 \mathrm{~b}$ ). Although individually small, these changes occurred repeatedly and consistently throughout the exon.

As the receptor-deficient maternal environment during the prenatal period is necessary and sufficient to elicit the anxietylike phenotype, ${ }^{7}$ we tested if prenatal exposure alone can 
a

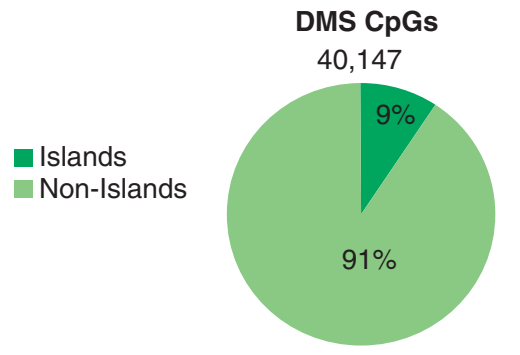

b

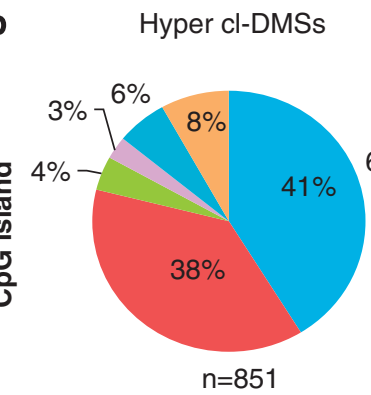

C

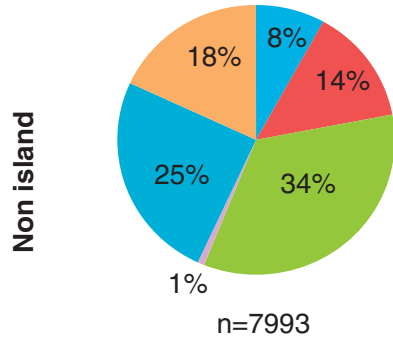

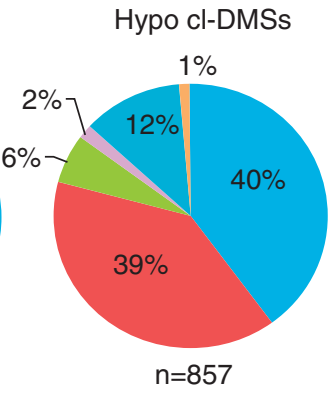

Hypo cl-DMSs

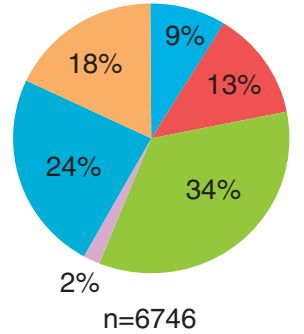

All CpGs

$1,740,400$
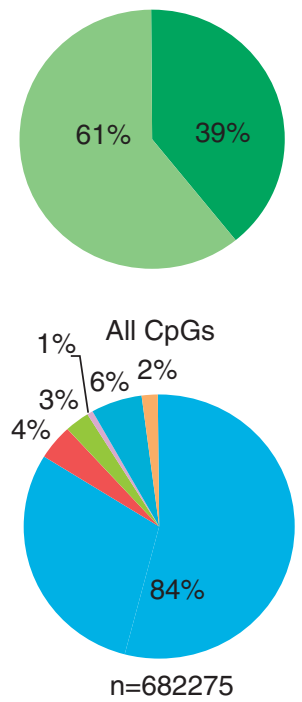

All CpGs

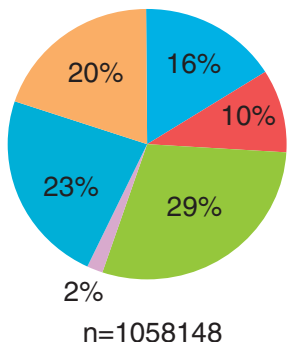

$\begin{array}{lll}\text { Promoter } & \text { Exon } \\ \text { Downstream } & \text { Intron } \\ \text { Distal } & \text { Intergenic }\end{array}$

Figure 3 Genomic features of differentially methylated sites in adverse maternal environment identified by enhanced reduced representation bisulfite sequencing (ERRBS). (a) CpG island and non-island-specific differentially methylated sites (DMSs) (WT(WT) vs WT(H) $+\mathrm{KO}(\mathrm{H})$ ). (b-c) Genomic features at clustered ( $<1 \mathrm{~kb})$ DMSs associated with $\mathrm{CpG}$ island and non-island sequences.

produce hypomethylation at the Atbf1 and Smo DMRs. WT offspring implanted as embryos into the oviducts of $\mathrm{KO}$ mothers and then cross-fostered at birth to WT mothers (referred to as WT(KO/WT) mice, Figure 2c) showed hypomethylation within both DMRs (Figure 2d). This indicates that exposure to the adverse maternal environment that is limited to the prenatal period is sufficient to elicit not only the anxiety phenotype but also the DNA methylation changes in the offspring.

Genome-wide differential methylation in adverse maternal environment. We used ERRBS to explore differential methylation at a larger fraction of $\mathrm{CpGs}$ and which is not limited to a predetermined set of $\mathrm{CpG}$ sites. ${ }^{29} \mathrm{~A}$ total of 376016818 aligned sequence reads of 51 bases were obtained from WT(WT), WT(H) and $K O(H)$ v-DGC DNA, which, at $\geqslant 35 \mathrm{X}$ coverage, reported methylation rates (MR, fraction of methylated cytosines at a site) at $1740900 \mathrm{CpG}$ sites $\left(8.4 \%\right.$ of all $\mathrm{CpGs}$ in the mouse) ${ }^{24}$ across the three groups of offspring. We found $2.3 \%$ of the $\mathrm{CpG}$ sites to be differentially methylated in both $W T(H)$ and $K O(H)$ neurons compared with WT(WT) neurons (BH-FDR $q<0.01$; $\Delta M R \geqslant 20 \%$; Figure $3 a$ ). Island-specific differentially methylated sites (DMSs) were underrepresented among all DMSs. This finding is in agreement with previous reports showing that islands are typically resistant to methylation; however, if methylated during development, islands usually are not subjected to tissue-specific or environmentally induced methylation changes. ${ }^{20,23,35}$

Close to half of the DMSs identified in adult DGCs (16447) were spaced $<1 \mathrm{~kb}$ from each other (clustered-DMSs; Figures $3 \mathrm{~b}$ and $\mathrm{c}$ ) and $>50 \%$ of these ' $<1 \mathrm{~kb}$ ' sites had an inter DMS distance between 2 and $100 \mathrm{bps}$ indicating a significant clustering of methylation changes (Supplementary Figure 2). $\mathrm{Cl}$-DMSs specified a total of 6357 DMRs. ERRBS also detected 4187 differentially methylated clustered sites when WT $(\mathrm{H})$ and $\mathrm{KO}(\mathrm{H})$ samples were compared indicating that the offspring genotype can elicit methylation changes as well. However, this smaller set of sites was not studied further because of lack of the association with the anxiety phenotype (Figure 1a). 


\section{All genomic CpGs}
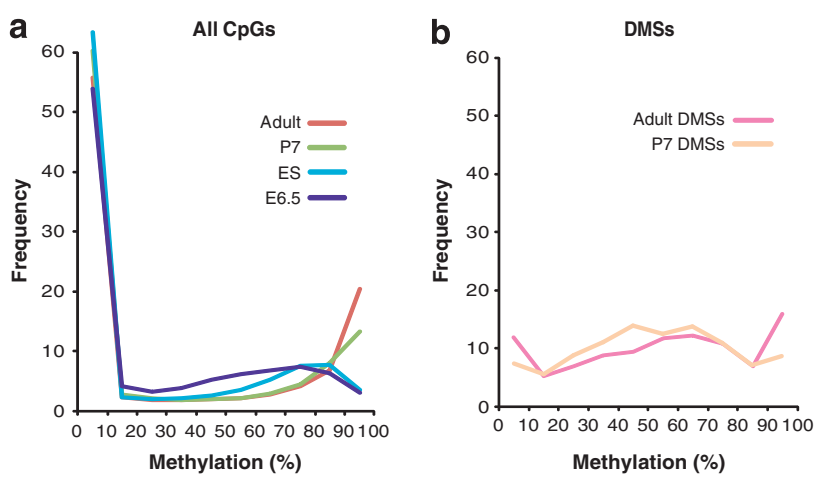

\section{Gene Body CpGs}
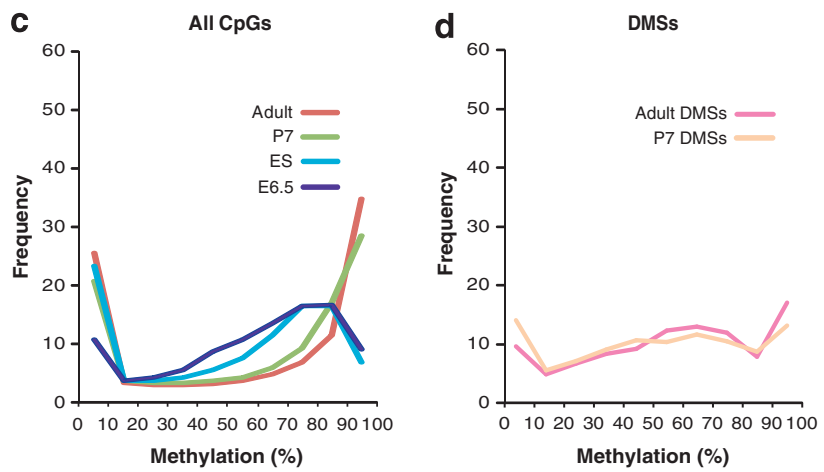

e

Type I

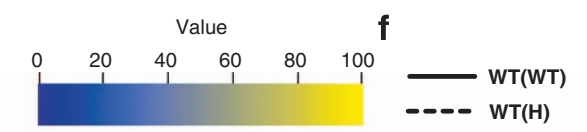

Type II
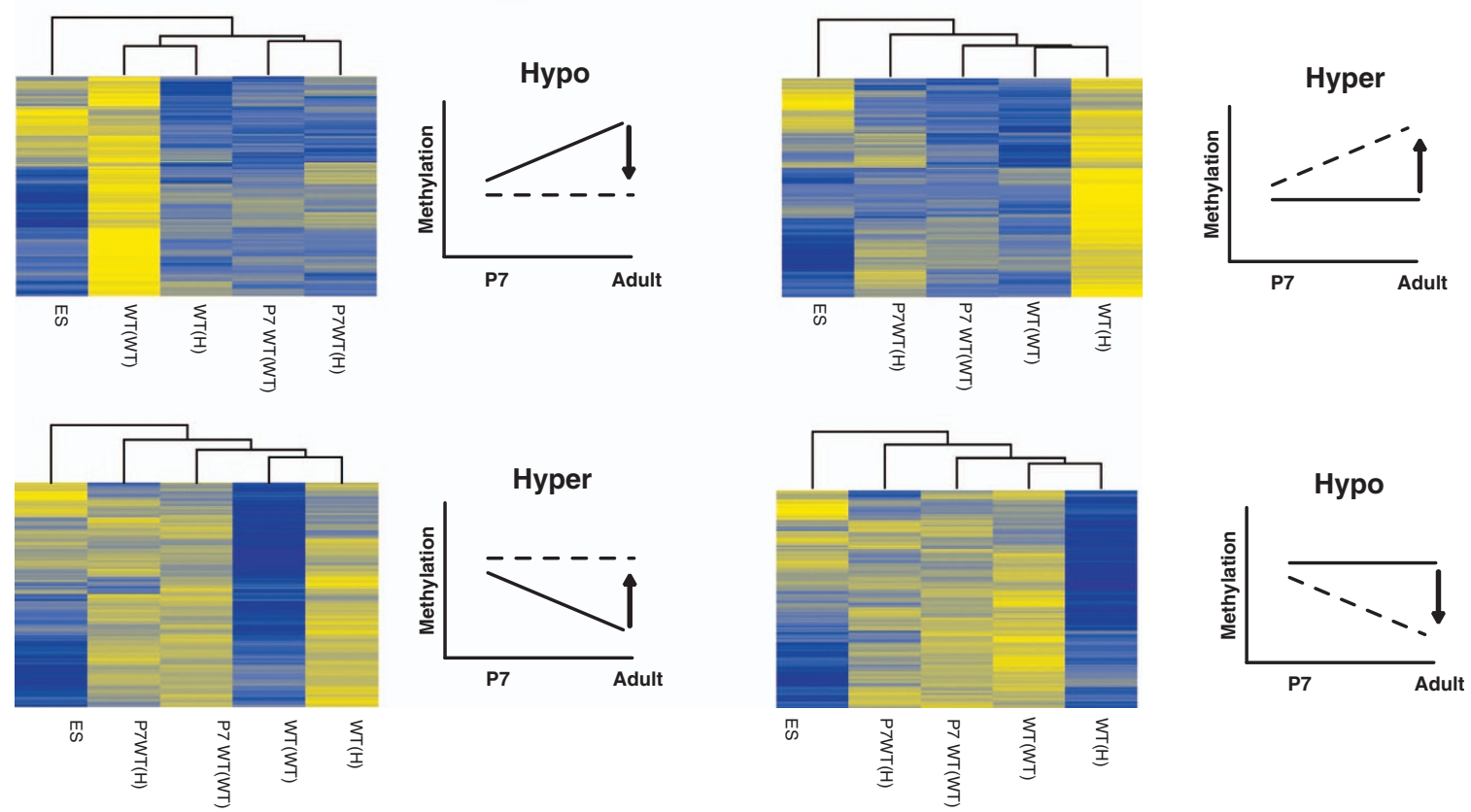

g
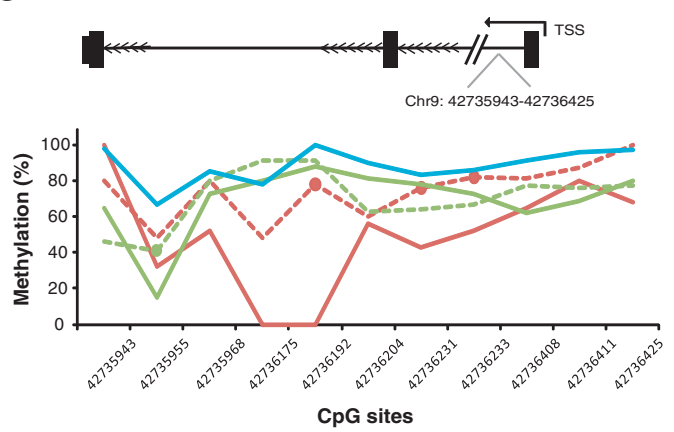

h
Bsn

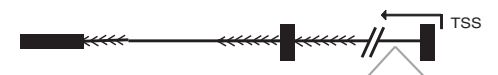

Chr9: 108082538-108086325

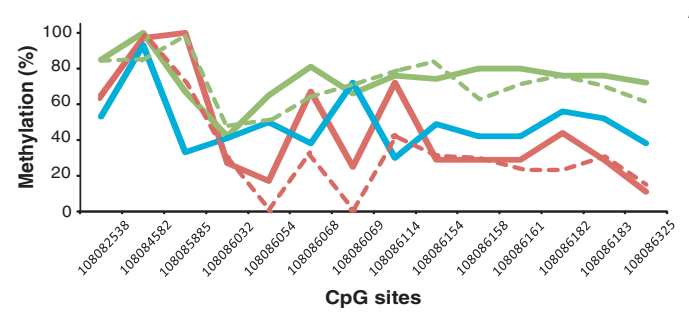

Adult-WTWT Adult-WTH $=-\infty$ P7- WTWT P7-WTH $-\infty$

ES

Figure 4 Methylation at differentially methylated sites (DMSs) during development in normal and adverse maternal environment. (a, b) Histograms with the distribution (\% of total) of methylation levels at all CpG sites and DMSs in 10\% bins in adult and P7 wild-type (WT) offspring of WT mothers (WT(WT)) dentate granule cells (DGCs), E6.5 epiblasts (E6.5) and embryonic stem (ES) cells. (c, d) Same as in AB but with gene body CpGs. (e, f) Methylation in P7 and adult DGCs in normal and adverse maternal environment at type I and type II hypo/ hypermethylated DMSs. Hierarchical clustering within the four groups of DMSs shows similar methylation levels at P7 in normal and adverse environment and type I and II specific changes in adults. Center: schematic representation of developmental changes at the four classes of DMSs from P7 to adult age; continuous and dashed lines represent changes in WT and $\mathrm{H}$ maternal environment, respectively. (g) A representative differentially methylated region (DMR) with type I hyper DMSs in Girk4. (h) A representative DMR with type II hypo DMSs in Bsn1. 

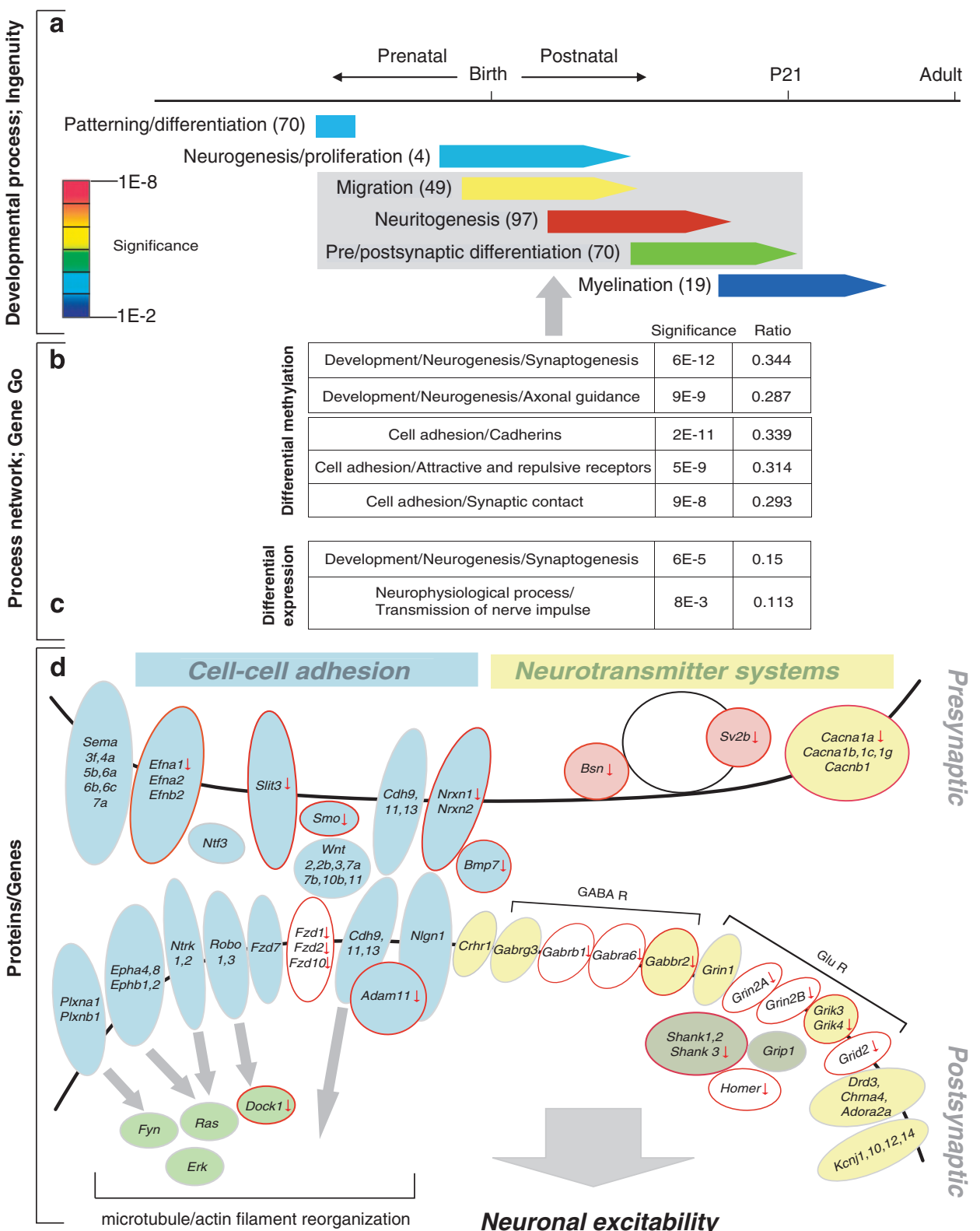

Figure 5 Differentially methylated genes encode synaptic proteins. (a, b) Ingenuity and GeneGo Process Network analysis of differentially methylated sites (DMSs) identifies synaptic functions. For the Ingenuity analysis, right-tailed Fisher's exact test was used to calculate a $P$-value determining the probability that each biological function assigned to the data set is due to chance alone. For the GeneGo analysis, statistical significance of a process was determined using the hypergeometric distribution and adjusted for multiple testing by MetaCore. Ratio represents the differentially methylated vs all genes in the functional categories. (c) GeneGo Process Network analysis of differentially expressed genes. (d) Differentially methylated genes encode adhesion molecules (blue) and their effectors (green), presynaptic proteins (pink), scaffolding proteins (gray) and neurotransmitter receptors (yellow). The figure positions the differentially methylated genes/proteins to the pre and postsynaptic compartments, also indicating those that interact with synaptic vesicles within the presynaptic compartment. The effect of cell adhesion molecules converge on the regulation of microtubule and actin organization that are essential in migration, neuritogenesis and synaptic differentiation, identified by functional analysis (panels a, b). Cell adhesion molecules are also involved in the recruitment of synaptic receptors whose function controls neuronal excitability. Genes for both inhibitory and excitatory receptors were among those that were differentially methylated. Some of the differentially methylated genes were also downregulated in adult dentate granule cells (DGCs) (red outline and arrow). Other synaptic genes were also downregulated but were not differentially methylated (white background with red outline and arrow). These data indicate a permanent hypofunction at many synaptic genes suggesting a persistent abnormality in synaptic plasticity and neuronal excitability.

The maternally specified cl-DMSs were further analyzed for their genomic features. ${ }^{36} \mathrm{CpG}$ island-associated DMSs, whether hypo- or hypermethylated, were found more often in exons $(\sim 10 \times$ enrichment, compared with all CpGs in islands) and less frequently in promoters (Figure $3 b$ ). These findings are consistent with results of the HELP assay that identified DMRs within exons (RRBs also identified the Atbf1 and Smo DMRs). The larger group of non-island DMSs (91\%) showed a slight enrichment for intronic and exonic CpGs $(34 \%$ vs $29 \%$ and $13 \%-14 \%$ vs $10 \%$, respectively), whereas 
promoter CpGs were underrepresented in cl-DMSs, at both hypo- and hypermethylated sites (16\% vs $8 \%-9 \%)$ (Figure 3c). Overall, these data indicate some preference of the maternal effect to modulate methylation at gene body CpGs, at both island and non-island sequences.

CpG methylation in the developing dentate gyrus in normal and adverse maternal environment. Majority of adult DGCs are generated during the perinatal and early postnatal period, followed by the gradual maturation of newly born cells. ${ }^{37}$ In the maternal $5 \mathrm{HT}_{1 \mathrm{~A}} \mathrm{R}$-deficient model, we observed normal proliferation but delayed neuronal maturation at the end of the first week of postnatal life, suggesting that young neurons may already have some epigenetic, either CpG methylation or chromatin, changes. To test this hypothesis, we profiled P7 neurons with ERRBS using the same statistical criteria and parameters that were used for the analysis of adult DGCs. CpG methylation in both P7 and adult neurons showed the typical bimodal distribution where the majority of $\mathrm{CpG}$ sites are either highly methylated $(>90 \%)$ or methylated at a low level $(<10 \%)$. However, the fraction of highly methylated CpG sites in P7 was slightly lower indicating ongoing methylation in young neurons (Figure 4a). As many DMSs mapped to introns and exons, the distribution of gene body CpGs was also assessed and showed a similar pattern (Figure 4c). This analysis also included E6.5 epiblasts (low coverage; from Smith et al. ${ }^{38}$ ) and embryonic stem (ES) cells (high coverage, this study) as references because epiblasts represent the last cell type with multipotency before the onset of gastrulation and cultured ES cells have an epiblast-like methylome. ${ }^{39}$ In agreement with previous reports, the distribution of methylated CpGs in epiblasts and ES cells indicated an incompletely methylated genome. ${ }^{38,40}$

In contrast to the bimodal distribution of methylation at all CpGs, methylation at clustered DMSs (Figures $3 b$ and c) was mostly intermediate in both P7 and adult DGCs (in normal maternal environment) and overall methylation levels were even lower than in epiblasts and ES cells (Figures $4 b$ and d). These data indicate that methylation at environmentally sensitive sites, in contrast to most CpG sites, is typically maintained in the intermediate range up to $\mathrm{P} 7$ and beyond. However, a closer inspection revealed significant rearrangements in the methylation of DMSs during development in normal and adverse environment (from P7 to adult). One group of DMSs showed a gain or loss of methylation during normal postnatal development, changes that were inhibited by maternal adversity (Figure $4 \mathrm{e}$ and Supplementary Figure 3). These 'type I DMSs' were divided into hypo- and hypermethylated subgroups according to the direction of the change in adversity. Sixty percent of DMSs belonged to these categories (33\% and $27 \%$ for hyper and hypo, respectively). Figures $2 b$ and $4 \mathrm{~g}$ illustrate clusters of type I hypo- and hypermethylated DMSs within the Atbf1/Smo and the Grik 4 genes, respectively. Methylation at 'type II DMSs' was not significantly changed during normal postnatal development but the $\mathrm{H}$ maternal environment resulted in hyper- or hypomethylation (Figure $4 \mathrm{f}$ and Supplementary Figure 3). A total of $40 \%(20 \%$ hypo and $20 \%$ hyper) of DMSs were type II. Typical type II hypomethylated DMSs are shown in the Bsngene (Figure 4h).
Although methylation at $\mathrm{CpG}$ sites is typically higher in P7 DGCs than in ES cells (and epiblasts; Figures 4a and c), methylation at DMSs in these cell types was similar (Figures $4 e$ and $f$ and Supplementary Figure 3), suggesting that no significant methylation changes occur at these sites in DGCs, either in normal or adverse environment, before their maturation (that is, before P7). Thus, the maternal effect, although prenatal in its origin, does not have an impact on methylation at DMSs until neurons develop beyond the young neuronal stage. Although type I and II hypo- and hypermethylated DMSs are quite different in terms of their methylation behavior in adverse maternal environment, we found no obvious sequence context or genomic features that would be group specific or predict the direction of their methylation. Overall, both arrest in developmental methylation/demethylation and abnormal gain/loss of methylation seem to account for producing differential methylation at specific sites in adversity.

Clusters of DMSs map to cell adhesion molecules and neurotransmitter receptor genes. We used the Ingenuity Knowledge Database to identify genes with relevant biological functions in the list of 1176 Ensembl genes harboring DMRs. A total of 510 genes belonged to the category of 'nervous system development and function' and could be mapped to major steps in neuronal development (Figure 5a). Genes with multiple functions were assigned to multiple developmental processes. This analysis identified neuritogenesis (103 genes with DMRs), migration (61 genes) and pre/postsynaptic differentiation (81 genes) as developmental processes highly enriched in differentially methylated genes. Neuritogenesis, the highest scoring process, is the growth and extension of neurites from the soma by precise cytoskeletal and adhesion dynamics, and guided by external attractive and repulsive cues. Migration and synaptic differentiation are tightly linked to neuritogenesis both in timing and shared molecules.

An independent analysis of the differentially methylated genes by GeneGo MetaCore Process Network Analysis identified essentially the same genes grouped to the functional networks of 'synaptogenesis', 'axonal guidance' and three 'cell adhesion' related clusters, 'cadherins', 'attractive and repulsive receptors' and 'synaptic contact' $(F D R<0.05$; Figure 5b). Indeed, the 'synaptogenesis' GeneGo category contained synaptic scaffolding proteins and neurotransmitter receptors that were also identified by the Ingenuity 'pre/postsynaptic differentiation' functional category. Similarly, cell adhesion molecules were identified by the Ingenuity analysis in migration, neuritogenesis and pre/ postsynaptic differentiation. Although the genes within these categories were 4.3 times larger than the average gene (138278 vs $31959 \mathrm{bp}$ ) and therefore could preferentially harbor DMSs, not all of these large genes were differentially methylated. Indeed, 556 genes out of the total of 738 within the functional categories in Figure $5 b$ were not modified by methylation. The difference in size between modified and nonmodified genes was only 2-fold (224942 and 109909, respectively), which suggests that the differential methylation is not proportionate with gene size. Also, the larger size of genes within the functional categories is mostly due to introns, 
but more of the gene-associated DMRs (52\% of total) were outside of introns in distal sequences (23\%), exons (16\%), promoters $(11 \%)$, and downstream sequences $(2 \%)$ than in introns (31\%).

Gene expression analysis of adult DGCs from offspring of WT and $\mathrm{H}$ mothers by RNA-Seq showed no overall correlation between expression and methylation at the 3069 DMRcontaining genes, whether the DMRs mapped to promoters, exons, introns or distal sequences (Supplementary Figure 4). However, analysis of the 1189 differentially expressed genes ( $>1.3$ fold; $q<0.05$, Fisher's exact R, BH-FDR; of which 193 contained DMR) with GeneGo Process Network identified 'synaptogenesis' as the top network (Figure 5c). Essentially, the same genes were also identified in the 'transmission of nerve impulse' network.

Overall, these functional analyses identified two major groups of proteins; cell adhesion molecules and neurotransmitter receptors (Figure 5d). Cell adhesion molecules have pre- and postsynaptic partners, and genes encoding both of these classes were differentially methylated. These included ephrins (EFNA1, A2, B2) and their receptors (EPHA4, A8, B1, B2), semaphorins (SEMA3F, 4A, 5B, 6A, 6B, 6C, 7A) and their plexin receptors (PLXNA1, B1), neurexin (NRXN1, 2) and neuroligin (NLGN1), Wnts (WNT2, 2b, 3, 7a, 7b, 10b, 11) and frizzled (FZD7), cadherins (CDH9, 11, 13) and slit (SLIT3) and its receptors (ROBO1, 3). Neurotransmission-related differentially methylated genes included those that encode neurotrophin 3 (NT3), the TrkA receptor (TRK1), $\mathrm{CRH}$ receptor 1 (CRHR1), GABA-B receptor subunit 2 (GABBR2), an NR1 subunit of the NMDA receptor (encoded by Grin1), the dopamine D3 receptor (DRD3), acetylcholine alpha 4 subunit (CHRNA4), adenosine A2 receptor (ADORA2A), a number of calcium channels (CACNA1A, 1C, 1G, B1) and K channels (KCNJ1, 10, 12, 14), which all have been implicated in neuronal excitability as well as in anxiety. ${ }^{41} \mathrm{~A}$ total of 18 genes within the functional clusters shown in Figures $5 \mathrm{a}$ and $\mathrm{b}$ were also differentially expressed (Supplementary Table 2; see 11 genes in Figure 5d) and almost exclusively downregulated (17 of 18) suggesting hypofunction at these genes. Additional synaptic genes that showed no differential methylation were downregulated, including those that encode the NR2A and 2B NMDA subunits (Grin2a and Grin2b), HOMER and FZD1, 2 and 10 (Figure 5d). Overall, the differential methylation/ expression of a large number of pre- and postsynaptic cell adhesion molecule and neurotransmitter receptor genes suggests a wide-ranging and permanent effect of the adverse maternal environment on synaptic plasticity and neuronal excitability.

\section{Discussion}

Pre and early postnatal adversity is a major factor in the development of psychiatric conditions. Offspring development is dependent on the maternal environment during these periods and numerous human and animal studies demonstrate that abnormal maternal physiology and behavior, whether genetic or environmental in nature, result in disruptions in normal brain development, which in turn can result in adolescent and adult behavioral abnormalities. ${ }^{11,42-44}$
The development of the hippocampus is particularly sensitive to environmental disruptions, presumably because hippocampal neurons show remarkable structural and functional plasticity. ${ }^{45}$ The hippocampal circuit is involved in numerous behaviors including cognitive tasks, evaluation and termination of the stress responses, and emotional behavior. ${ }^{12}$ Although the consequences of early life adversity on the hippocampus are relatively well documented at the behavioral and even morphological level, $5,11,45$ little is known about the underlying molecular mechanisms. Development is programmed by both transcriptional and epigenetic mechanisms and here we studied the developmental dynamics of $\mathrm{CpG}$ methylation genome-wide in DGCs in normal and adverse maternal environment. We used a previously established maternal adversity paradigm that is based on a $5-\mathrm{HT}_{1 \mathrm{~A}} \mathrm{R}$ deficit in the mother and which produces a delay in the development of the v-DGCs as well as an anxiety-like behavior. $^{7}$

The main finding of our work is that, although the receptordeficient maternal environment had a genome-wide effect, DNA methylation changes occurred in specific genomic locations and contexts. Specifically, CpG sites that were targeted by the maternal effect tended to have an intermediate methylation level during neuronal development until P7 and even beyond compared with the majority of the genome that exhibited either high $(>90 \%)$ or low $(<10 \%)$ methylation levels early on. Indeed, methylation at DMSs in developing DGCs was similar or even less than in ES cell or epiblasts, cells that exhibit extensive epigenetic plasticity ${ }^{40}$ and we speculate that epigenetic metastability at DMSs explains their sensitivity to disruptions by maternal adversity. Although intermediate methylation can be due to allelic differences documented at dosage compensated and imprinted genes, partial methylation in hematopoietic cells was predominantly associated with stochastic variability in methylation. ${ }^{46}$ As the vast majority of DMSs were not at imprinted genes, and because partial methylation was also seen in males at the $X$ chromosome, we believe that the intermediate methylation at most DMSs is stochastic in nature.

The maternal effect either modified the developmental trajectory of methylation at DMSs by inhibiting programmed hypo/hypermethylation (type I DMSs) or induced abnormal hypo/hypermethylation at sites that normally stay unchanged during postnatal development (type II DMSs). These influences produced relative hypo- and hypermethylation in the $\mathrm{H}$ maternal as compared with the WT maternal environment in DGCs. This suggests different mechanisms for the hypo- and hypermethylation and further studies will be needed to determine the factors responsible for the direction of methylation change in adverse maternal environment. As ERRBS typically profiles one strand, it was not possible to determine if gain in methylation during development, or by the maternal effect at DMSs, was due to de novo methylation or maintenance methylation at asymmetrically methylated sites. On the other hand, loss of methylation at DMSs is likely due to active rather than passive demethylation because in the postnatal dentate gyrus the majority of isolated DGCs are postmitotic and because the number of neuronal precursors, located in the subgranular layer, and glial cells is too low to 
significantly contribute to the overall signal. Several mechanisms have been shown to account for active demethylation including oxidative demethylation of cytosines achieved by the ten-eleven-translocation proteins and repair-based mechanisms. ${ }^{47}$

DMSs are relatively scarce at promoters and typically found in gene bodies and gene distal areas. This suggests that the maternal effect may not regulate transcription via proximal promoters, but may rather influences expression indirectly by modulating alternative splicing and promoter use, and/or miRNAs expression. Further analysis showed that clusters of DMSs, that is, DMRs, were enriched in genes that encode proteins involved in adhesion molecules and neurotransmitter receptors. Up to $34 \%$ of the genes classified in these categories were differentially methylated suggesting a strong convergence of the maternal effect on these genes and functions. Gene expression studies identified some of the differentially methylated genes and overall differential methylation and expression showed a good correspondence.

It is challenging to establish a causative relationship between the DNA methylation changes in DGCs and innate anxiety, the main phenotype of the offspring of receptor mutant mothers, ${ }^{9}$ because it is expected that each individual differentially methylated gene contributes only a small fraction of the total phenotype. The association of the differentially methylated genes to the behavioral phenotype is likely similar to that of susceptibility genes to polygenic diseases/conditions. Network and functional analyses have been developed for these situations and we employed two computational models that use different algorithms to identify putative functionally relevant differentially methylated genes. The validity of our approach is strengthened by the fact that many of the differentially methylated/expressed genes and their protein products have individually been linked to anxiety in human or to anxiety-like behavior in animals by pharmacological and/or genetic evidence. The genes for the neurotransmitter receptors/subunits CRHR1, DRD3, ADORA2A, CHRNA4, GABRG3 and GABBR2 were differentially methylated and all of these receptors have been linked to anxiety. Central administration of $\mathrm{CRH}$ in rodents produces behavioral effects via CRHR1/2 that correlate with a state of anxiety, such as a reduction in exploration in a novel environment or an enhanced fear response. ${ }^{48}$ Also, genetic studies indicate that CRHR1 is important in regulating anxiety levels. ${ }^{49,50}$ DRD3 knockout mice display reduced anxiety in the open field and elevated plus maze associated with increased locomotor activity. ${ }^{51}$ Adenosine $_{2 a}$ receptor (ADORA2A) null mice show increased anxiety in elevated maze and light dark box. ${ }^{52}$ Mice null for the Ach receptor $\alpha_{4}$ subunit (CHRNA4), display an increased anxiety in the elevated plus maze test. ${ }^{53}$ Inhibitory GABA-A receptors are central in the treatment of anxiety and receptor deficit in the hippocampus and parahippocampus has also been implicated in panic disorder and generalized anxiety disorder. ${ }^{54-56}$ Similarly, GABRB2 KO mouse exhibit anxiety. ${ }^{57}$ Increased excitability is another mechanism that can lead to anxiety. Differential methylation was detected in Grin 1, the gene for the NMDA NR1 subunit, in Grik3 and Grik4, the genes for KA receptor subunits, in genes for glutamate receptor interacting proteins such as Grip1, and Shank1, 2, 3 as well as in calcium (Cacna1c) and potassium channel (Kcnj) genes that can all influence neuronal excitability and modulate anxiety. ${ }^{58-61}$

Cell adhesion molecules, comprising the other large group of genes with differential methylation, are utilized through many steps during CNS development, including neuronal migration, guidance, neurite outgrowth and synapse formation. We detected differential methylation at genes encoding cadherins, ephrins, slit-robo, wnts, semaphorins-plexins and neurexin-neuroligins (see Figure 5). Disruptions of these genes typically result in neurodevelopmental conditions with a wide variety of symptom including cognitive defects, anxiety and abnormal social behavior. Mutations in some of these genes such as $C D H 9$ and NRNX1 in human, which were differentially methylated in the presence of adverse maternal environment in mouse, have been implicated in autism spectrum disorders and schizophrenia. ${ }^{62-65}$ Specifically, loss of function mutations (deletion and copy number variation) in $N R X N 1$, a gene that was not only differentially methylated but also downregulated in our experiments, have been linked to a number but individually variable autism spectrum disorder symptoms within affected family members resulting in relatively selective behavioral abnormalities such as impaired social interactions, anxiety, or learning and memory deficits in individuals. $^{62,63,66}$ Another example of a relatively specific anxiety phenotype associated with an adhesion molecule is the increased avoidance of the open arm of mice with altered EPHB2, an ephrin receptor whose gene was also differentially methylated in the receptor-deficient maternal environment. ${ }^{67}$ Overall, our finding of differential methylation/expression at a large number of cell adhesion and neurotransmitter receptor genes suggests that some forms of anxiety following maternal adversity could be associated with epigenetic perturbations in multiple synaptic genes, each contributing only a small effect to the overall phenotype.

\section{Conflict of interest}

The authors declare no conflict of interest.

Acknowledgements. We thank Yushan Li of the Epigenomics core facility and Dr Jenny Xiang of the Genomics Resources Core Facility for technical support. We also thank Dr Piali Mukherjee for computational analysis assistance and Drs Maria Figueroa, Min seok Song and Sung hyun Kim for technical advice. This work was supported by US National Institute of Mental Health grant 5RO1MH086883 and 1R01MH58669 to MT.

Raw data (HELP, RNA-Seq and RRBS) have been deposited into the GEO database and are included in the super series GSE35856. The following link has been created to allow review of record GSE35815: http://www.ncbi.nlm.nih.gov/geo/ query/acc.cgi?token=rbwjncouicamlc\&acc=GSE35856

1. Gilbert R, Widom CS, Browne K, Fergusson D, Webb E, Janson S. Burden and consequences of child maltreatment in high-income countries. Lancet 2009; 373: 68-81.

2. Green JG, McLaughlin KA, Berglund PA, Gruber MJ, Sampson NA, Zaslavsky AM et al. Childhood adversities and adult psychiatric disorders in the national comorbidity survey replication I: associations with first onset of DSM-IV disorders. Arch Gen Psychiatry 2010; 67: $113-123$

3. Chen GH, Wang H, Yang QG, Tao F, Wang C, Xu DX. Acceleration of age-related learning and memory decline in middle-aged CD-1 mice due to maternal exposure to ipopolysaccharide during late pregnancy. Behav Brain Res 2011; 218: 267-279.

4. Veenema AH, Reber SO, Selch S, Obermeier F, Neumann ID. Early life stress enhances the vulnerability to chronic psychosocial stress and experimental colitis in adult mice. Endocrinology 2008; 149: 2727-2736. 
5. Millstein RA, Holmes A. Effects of repeated maternal separation on anxiety- and depression-related phenotypes in different mouse strains. Neurosci Biobehav Rev 2007; 31: 3-17.

6. Priebe K, Romeo RD, Francis DD, Sisti HM, Mueller A, McEwen BS et al. Maternal influences on adult stress and anxiety-like behavior in C57BL/6J and BALB/CJ mice: a cross-fostering study. Dev Psychobiol 2005; 47: 398-407.

7. Gleason G, Liu B, Bruening S, Zupan B, Auerbach A, Mark W et al. The serotonin1A receptor gene as a genetic and prenatal maternal environmental factor in anxiety. Proc Natl Acad Sci USA 2010; 107: 7592-7597.

8. van Velzen A, Toth M. Role of maternal $5-\mathrm{HT}(1 \mathrm{~A})$ receptor in programming offspring emotional and physical development. Genes Brain Behav 2010; 9: 877-885.

9. Gleason G, Zupan B, Toth M. Maternal genetic mutations as gestational and early life influences in producing psychiatric disease-like phenotypes in mice. Front Psychiatry/Front Res Foundation 2011; 2: 25.

10. Zoghbi HY, Warren ST. Neurogenetics: advancing the 'next-generation' of brain research Neuron 2010; 68: 165-173.

11. Meaney MJ. Maternal care, gene expression, and the transmission of individual differences in stress reactivity across generations. Annu Rev Neurosci 2001; 24: 1161-1192.

12. Bannerman DM, Rawlins JN, McHugh SB, Deacon RM, Yee BK, Bast T et al. Regional dissociations within the hippocampus-memory and anxiety. Neurosci Biobehav Rev 2004; 28: 273-283.

13. Kjelstrup KG, Tuvnes FA, Steffenach HA, Murison R, Moser El, Moser MB. Reduced fea expression after lesions of the ventral hippocampus. Proc Natl Acad Sci USA 2002; 99 10825-10830.

14. Adhikari A, Topiwala MA, Gordon JA. Synchronized activity between the ventral hippocampus and the medial prefrontal cortex during anxiety. Neuron 2010; 65: 257-269.

15. Ishikawa A, Nakamura S. Ventral hippocampal neurons project axons simultaneously to the medial prefrontal cortex and amygdala in the rat. $J$ Neurophysiol 2006; 96: 2134-2138.

16. Elliott E, Ezra-Nevo G, Regev L, Neufeld-Cohen A, Chen A. Resilience to social stress coincides with functional DNA methylation of the Crf gene in adult mice. Nat Neurosci2010; 13: $1351-1353$

17. Oberlander TF, Weinberg J, Papsdorf M, Grunau R, Misri S, Devlin AM. Prenatal exposure to maternal depression, neonatal methylation of human glucocorticoid receptor gene (NR3C1) and infant cortisol stress responses. Epigenetics 2008; 3: 97-106.

18. Zhang TY, Hellstrom IC, Bagot RC, Wen X, Diorio J, Meaney MJ. Maternal care and DNA methylation of a glutamic acid decarboxylase 1 promoter in rat hippocampus. $J$ Neurosci 2010; 30: 13130-13137.

19. McGowan PO, Sasaki A, D’Alessio AC, Dymov S, Labonte B, Szyf M et al. Epigenetic regulation of the glucocorticoid receptor in human brain associates with childhood abuse. Nat Neurosci 2009; 12: 342-348.

20. Guo JU, Ma DK, Mo H, Ball MP, Jang MH, Bonaguidi MA et al. Neuronal activity modifies the DNA methylation landscape in the adult brain. Nat Neurosci 2011; 14: 1345-1351.

21. Rollins RA, Haghighi F, Edwards JR, Das R, Zhang MQ, Ju J et al. Large-scale structure of genomic methylation patterns. Genome Res 2006; 16: 157-163.

22. Khulan B, Thompson RF, Ye K, Fazzari MJ, Suzuki M, Stasiek E et al. Comparative isoschizomer profiling of cytosine methylation: the HELP assay. Genome Res 2006; 16 : 1046-1055.

23. Mohn F, Weber M, Rebhan M, Roloff TC, Richter J, Stadler MB et al. Lineage-specific polycomb targets and de novo DNA methylation define restriction and potential of neuronal progenitors. Mol Cell 2008; 30: 755-766.

24. Meissner A, Mikkelsen TS, Gu H, Wernig M, Hanna J, Sivachenko A et al. Genome-scale DNA methylation maps of pluripotent and differentiated cells. Nature 2008; 454: 766-770.

25. Murgatroyd C, Patchev AV, Wu Y, Micale V, Bockmuhl Y, Fischer D et al. Dynamic DNA methylation programs persistent adverse effects of early-life stress. Nat Neurosci 2009; 12: 1559-1566.

26. Figueroa ME, Melnick A, Greally JM. Genome-wide determination of DNA methylation by Hpa II tiny fragment enrichment by ligation-mediated PCR (HELP) for the study of acute leukemias. Methods Mol Biol 2009; 538: 395-407.

27. Figueroa ME, Reimers M, Thompson RF, Ye K, Li Y, Selzer RR et al. An integrative genomic and epigenomic approach for the study of transcriptional regulation. PLOS ONE 2008; 3: e1882.

28. Ehrich M, Nelson MR, Stanssens P, Zabeau M, Liloglou T, Xinarianos G et al. Quantitative high-throughput analysis of DNA methylation patterns by base-specific cleavage and mass spectrometry. Proc Nat Acad Sci USA 2005; 102: 15785-15790.

29. Gu H, Smith ZD, Bock C, Boyle P, Gnirke A, Meissner A. Preparation of reduced representation bisulfite sequencing libraries for genome-scale DNA methylation profiling Nat Protoc 2011; 6: 468-481.

30. Kielbasa SM, Wan R, Sato K, Horton P, Frith MC. Adaptive seeds tame genomic sequence comparison. Genome Res 2011; 21: 487-493.

31. Qi Y, Ranish JA, Zhu X, Krones A, Zhang J, Aebersold R et al. Atbf1 is required for the Pit1 gene early activation. Proc Nat Acad Sci USA 2008; 105: 2481-2486.

32. Jung CG, Kim HJ, Kawaguchi M, Khanna KK, Hida H, Asai K et al. Homeotic factor ATBF1 induces the cell cycle arrest associated with neuronal differentiation. Development (Cambridge, England) 2005; 132: 5137-5145.
33. Machold R, Hayashi S, Rutlin M, Muzumdar MD, Nery S, Corbin JG et al. Sonic hedgehog is required for progenitor cell maintenance in telencephalic stem cell niches. Neuron 2003; 39: 937-950.

34. Sanchez-Camacho C, Bovolenta P. Emerging mechanisms in morphogen-mediated axon guidance. Bioessays 2009; 31: 1013-1025.

35. Irizarry RA, Ladd-Acosta C, Wen B, Wu Z, Montano C, Onyango P et al. The human colon cancer methylome shows similar hypo- and hypermethylation at conserved tissue-specific $\mathrm{CpG}$ island shores. Nat Genet 2009; 41: 178-186.

36. Giannopoulou EG, Elemento O. An integrated ChIP-seq analysis platform with customizable workflows. BMC Bioinformatics 2011; 12: 277

37. Muramatsu R, Ikegaya $\mathrm{Y}$, Matsuki $\mathrm{N}$, Koyama R. Neonatally born granule cells numerically dominate adult mice dentate gyrus. Neuroscience 2007; 148: 593-598.

38. Smith ZD, Chan MM, Mikkelsen TS, Gu H, Gnirke A, Regev A et al. A unique regulatory phase of DNA methylation in the early mammalian embryo. Nature 2012; 484: 339-344.

39. Borgel J, Guibert S, Li Y, Chiba H, Schubeler D, Sasaki H et al. Targets and dynamics of promoter DNA methylation during early mouse development. Nat Genet 2010; 42: 1093-1100.

40. Lister R, Pelizzola M, Dowen RH, Hawkins RD, Hon G, Tonti-Filippini J et al. Human DNA methylomes at base resolution show widespread epigenomic differences. Nature 2009; 462: 315-322.

41. Wood SJ, Toth M. Molecular pathways of anxiety revealed by knockout mice. $\mathrm{Mol}$ Neurobiol 2001; 23: 101-119.

42. Charil A, Laplante DP, Vaillancourt C, King S. Prenatal stress and brain development. Brain Res Rev 2010; 65: 56-79.

43. Phillips NK, Hammen CL, Brennan PA, Najman JM, Bor W. Early adversity and the prospective prediction of depressive and anxiety disorders in adolescents. J Abnorm Child Psychol 2005; 33: 13-24.

44. Nemeroff $C B$. Early-life adversity, CRF dysregulation, and vulnerability to mood and anxiety disorders. Psychopharmacol Bull 2004; 38(Suppl 1): 14-20.

45. McEwen BS. Stress and hippocampal plasticity. Annu Rev Neurosci 1999; 22: 105-122.

46. Hodges E, Molaro A, Dos Santos CO, Thekkat P, Song Q, Uren PJ et al. Directional DNA methylation changes and complex intermediate states accompany lineage specificity in the adult hematopoietic compartment. Mol Cell 2011; 44: 17-28.

47. Niehrs $C$, Schafer A. Active DNA demethylation by Gadd45 and DNA repair. $\operatorname{Tr}$ Cell Biol 2012; 22: 220-227.

48. Sutton RE, Koob GF, Le Moal M, Rivier J, Vale W. Corticotropin releasing factor produces behavioural activation in rats. Nature 1982; 297: 331-333.

49. Smith GW, Aubry JM, Dellu F, Contarino A, Bilezikjian LM, Gold LH et al. Corticotropin releasing factor receptor 1-deficient mice display decreased anxiety, impaired stress response, and aberrant neuroendocrine development. Neuron 1998; 20: 1093-1102.

50. Timpl P, Spanagel R, Sillaber I, Kresse A, Reul JM, Stalla GK et al. Impaired stress response and reduced anxiety in mice lacking a functional corticotropin-releasing hormone receptor 1. Nat Genet 1998; 19: 162-166.

51. Accili D, Fishburn CS, Drago J, Steiner H, Lachowicz JE, Park BH et al. A targeted mutation of the D3 dopamine receptor gene is associated with hyperactivity in mice. Proc Nat Acad Sci USA 1996; 93: 1945-1949.

52. Ledent C, Vaugeois JM, Schiffmann SN, Pedrazzini T, El Yacoubi M, Vanderhaeghen JJ et al. Aggressiveness, hypoalgesia and high blood pressure in mice lacking the adenosine A2a receptor. Nature 1997; 388: 674-678.

53. Ross SA, Wong JY, Clifford JJ, Kinsella A, Massalas JS, Horne MK et al. Phenotypic characterization of an alpha 4 neuronal nicotinic acetylcholine receptor subunit knock-out mouse. J Neurosci 2000; 20: 6431-6441.

54. Schlegel S, Steinert H, Bockisch A, Hahn K, Schloesser R, Benkert O. Decreased benzodiazepine receptor binding in panic disorder measured by IOMAZENIL-SPECT. A preliminary report. Eur Arch Psychiatry Clin Neurosci 1994; 244: 49-51.

55. Kaschka W, Feistel H, Ebert D. Reduced benzodiazepine receptor binding in panic disorders measured by iomazenil SPECT. J Psychiatr Res 1995; 29: 427-434.

56. Tiihonen J, Kuikka J, Rasanen P, Lepola U, Koponen H, Liuska A et al. Cerebral benzodiazepine receptor binding and distribution in generalized anxiety disorder: a fractal analysis. Mol Psychiatry 1997; 2: 463-471.

57. Mombereau C, Kaupmann K, Gassmann M, Bettler B, van der Putten H, Cryan JF. Altered anxiety and depression-related behaviour in mice lacking $\mathrm{GABAB}(2)$ receptor subunits. Neuroreport 2005; 16: 307-310.

58. Barkus C, McHugh SB, Sprengel R, Seeburg PH, Rawlins JN, Bannerman DM. Hippocampal NMDA receptors and anxiety: at the interface between cognition and emotion. Eur J Pharmacol 2010; 626: 49-56.

59. Lee AS, Ra S, Rajadhyaksha AM, Britt JK, De Jesus-Cortes H, Gonzales KL et al. Forebrain elimination of cacna1c mediates anxiety-like behavior in mice. Mol Psychiatry 2012; 17: 1054-5.

60. Dong Y, Green T, Saal D, Marie H, Neve R, Nestler EJ et al. CREB modulates excitability of nucleus accumbens neurons. Nat Neurosci 2006; 9: 475-477.

61. Catches JS, Xu J, Contractor A. Genetic ablation of the GluK4 kainate receptor subunit causes anxiolytic and antidepressant-like behavior in mice. Behav Brain Res 2012; 228: 406-414. 
62. Szatmari P, Paterson AD, Zwaigenbaum L, Roberts W, Brian J, Liu XQ et al. Mapping autism risk loci using genetic linkage and chromosomal rearrangements. Nat Genet 2007; 39: 319-328.

63. Kim HG, Kishikawa S, Higgins AW, Seong IS, Donovan DJ, Shen Y et al. Disruption of neurexin 1 associated with autism spectrum disorder. Am J Hum Genet 2008; 82 199-207.

64. Morrow EM, Yoo SY, Flavell SW, Kim TK, Lin Y, Hill RS et al. Identifying autism loci and genes by tracing recent shared ancestry. Science 2008; 321: 218-223.

65. Wang K, Zhang H, Ma D, Bucan M, Glessner JT, Abrahams BS et al. Common genetic variants on 5p14.1 associate with autism spectrum disorders. Nature 2009; 459: 528-533.

66. Wisniowiecka-Kowalnik B, Nesteruk M, Peters SU, Xia Z, Cooper ML, Savage S et al. Intragenic rearrangements in NRXN1 in three families with autism spectrum disorder, developmental delay, and speech delay. Am J Med Genet B Neuropsychiatr Genet 2010; 153B: $983-993$

67. Attwood BK, Bourgognon JM, Patel S, Mucha M, Schiavon E, Skrzypiec AE et al. Neuropsin cleaves EphB2 in the amygdala to control anxiety. Nature 2011; 473: 372-375.

(c) Translational Psychiatry is an open-access journal SOMERIHWHS HESERVED published by Nature Publishing Group. This work is licensed under the Creative Commons Attribution-NonCommercial-No Derivative Works 3.0 Unported License. To view a copy of this license, visit http://creativecommons.org/licenses/by-nc-nd/3.0/

Supplementary Information accompanies the paper on the Translational Psychiatry website (http://www.nature.com/tp) 